\title{
Mathematical Model and Simulation of Austenite Reverse Phase Transformation Process in Cold Rolled Low Carbon Steel
}

\author{
Su-fen WANG, Zhi-jie LI*, Jian-hong HUANG, Xu-yang CHEN, Xin-dong WANG
}

\begin{abstract}
Based on the reverse austenite transformation process of cold rolled low carbon steel and the deformation energy storage, the mathematical model of phase transformation temperature and structure transformation of austenite reverse transformation was established by using Scheil transformation kinetics. The different heating temperature of austenite and deformation amount of the austenite reverse transformation structure are numerically simulated. The inverse transformation process of austenite was calculated by Matlab, the calculation results show that the austenite transformation temperature $A_{s}$ increases with the increase of heating rate and comes to a constant value. The influence of deformation on $A_{s}$ decreases with the increase of heating rate.Austenite grain size and the temperature is approximately exponential. The accuracy of the model was verified by experimental analysis of the microstructure under different process conditions.
\end{abstract}

Keywords: austenite; cold rolled low carbon steel; numerical model; reverse phase transformation; simulation

\section{INTRODUCTION}

Cold rolled low carbon steel is mainly used in automobiles, home appliances and other industries. Cold deformation and annealing processes affect the final structure and performance of the product, but its organization and control are mainly achieved by postrolling annealing. When annealed, heating to the $A c_{1}$ (the critical temperature of transformation when heating) temperature above the austenite reverse phase transformation and subsequent cooling occurs, a series of tissue transformation processes determine the final microstructure of the low carbon steel, which in turn determine product performance [1-3]. The annealing heat treatment of steel is composed of three stages: heating, heat preservation and cooling $[4,5]$, the microstructure evolution during annealing and heating austenite formation is a very complicated process. Deformation and heating conditions have an important influence on the final austenite grain size, morphology, crystal orientation and internal structure, all of which directly affect the final structure and properties of the cooling process.

Based on the Scheil phase transition kinetics theory, considering the deformation energy storage, the mathematical model of the austenite reverse transformation process of cold rolled low carbon steel annealing was established. The phase transition temperature was calculated, and the influence of deformation and temperature on the microstructure of austenite was analyzed. The accuracy of the model was verified by simulation experiments.

\section{MATHEMATICAL MODEL}

\subsection{Phase Change Incubation Model}

For the continuous phase transition process, when the temperature is at the thermodynamic equilibrium temperature, the key factors that affect the phase change are whether the incubation period can meet the requirements. The phase change incubation period is a continuous accumulation process. The superposition rule proposed by Scheil is used to calculate the incubation period of the continuous cooling or heating proces, and the continuous cooling phase transformation is processed into the sum of the micro isothermal phase transitions [6-8]. During continuous cooling (heating), when $t=t_{n}$ (corresponding to temperature $T_{n}$ ), the sum of the incubation periods required for each temperature stop is 1 , then the phase transition begins, which can be expressed as:

$\sum_{i=1}^{i=n} \frac{\Delta t}{\tau_{i}}=1$

where $\Delta t$ is the residence time (s) at the temperature $T$ during the continuous phase transition and $\tau_{i}$ is the incubation period (s) at the temperature $T_{i}$.

Whether it is hot rolling or thermal simulation, the deformation increases the phase equilibrium temperature and shortens the phase change incubation period. According to the theory of deformation promoting phase transformation, deformation causes the internal lattice of the material to be distorted, and the dislocation density increases. The deformation storage energy in the body promotes the nucleation rate. Based on the above research, this paper considers the influence of deformation and establishes the phase change incubation model as $[9,10]$ :

$\tau=\frac{3\left(a_{\alpha}+a_{\gamma}\right)^{4} R T E_{\gamma}(1-\varepsilon)^{3 / 2}}{D_{C} x_{C}^{\alpha} v_{\gamma}{ }^{2} \Delta G_{A}{ }^{2}}$

where $v_{\gamma}$ is a function of austenite lattice parameter, the expression is $v_{\gamma}=a_{\gamma}^{3} / 2, \varepsilon$ is relative deformation. $E_{\gamma}$ is the interfacial energy of disordered austenite, $E_{\gamma}=0.503 \mathrm{~J} / \mathrm{m}^{2}$. Boltzmann constant $R=1.38 \times 10^{-23} \mathrm{~J} / \mathrm{K} . a_{\gamma}$ and $a_{\alpha}$ are the lattice parameters of austenite and ferrite, respectively, which can be expressed as

$$
\begin{aligned}
& a_{\alpha}=2.8863 \cdot\left[1+17.5 \times 10^{-6}(T-800)\right] \\
& a_{\gamma}=\left(3.6306+0.78 x_{C}^{\gamma}\right) . \\
& \cdot\left[1+\left(24.9-50 x_{C}^{\gamma}\right) \times 10^{-6} \cdot(T-1000)\right]
\end{aligned}
$$

$D_{C}$ is the diffusion coefficient of $\mathrm{C}$ in ferrite, which can 
be calculated according to Kaufman formula

$$
\left\{\begin{array}{l}
D_{C}=0.8 \exp \left(-19 x_{C}^{\alpha}\right) \exp \left(-\frac{Q_{d}}{R T}\right) \\
Q_{d}=25800-3.1 \times 10^{5} x_{C}^{\alpha}+4.2 \times 10^{5}\left(x_{C}^{\alpha}\right)^{2}
\end{array}\right.
$$

where $Q_{d}$ is the activation energy of carbon atom diffusion in ferrite.

\subsection{Austenite Phase Variable Model}

The phase change of the heating process is the sum of the micro-isothermal phase transitions. After the phase transition occurs, $\alpha \rightarrow \gamma$ satisfies the additivity rule, and the superposed phase transition amount is:

$X_{n}=X_{n-1}+\Delta X_{n}$

where $X_{n}$ is amount of transformation at the time $n, X_{n-1}$ is amount of transformation at the time $(n-1), \Delta X_{n}$ is amount of transformation from the time $(n-1)$ to the time $n$.

In the heating process, the phase change is carried out in the manner of nucleation growth [11]. With the change of time increment $\Delta t^{\langle i\rangle}$, the increment of phase transformation rate $\Delta X^{\langle i\rangle}$ can be expressed as:

$$
\begin{aligned}
& \Delta X_{A}^{\langle i\rangle}=4\left(\frac{\pi}{3}\right)^{1 / 4}\left(N_{\mathrm{A}}^{\langle i\rangle} S_{\alpha}\right)^{1 / 4}\left(G_{\mathrm{A}}^{\langle i\rangle}\right)^{3 / 4} \\
& \left(\ln \frac{1}{1-X_{A}^{\langle i-1\rangle}}\right)^{3 / 4}\left(1-X_{A}^{\langle i-1\rangle}\right) \Delta t^{\langle i\rangle}
\end{aligned}
$$

In the heat preservation phase, in line with the "position saturation" mechanism, the phase change rate increment can be expressed as:

$\Delta X_{A}^{\langle i\rangle}=K_{3} S_{\alpha} G_{A}^{\langle i\rangle}\left(1-X_{A}^{\langle i-1\rangle}\right) \Delta t^{\langle i\rangle}$

where $\Delta X_{A}^{\langle i\rangle}$ is the austenite volume fraction (\%) that has been transformed in the stage of $(i-1)$, and $S_{\alpha}$ is the effective grain boundary area $\left(\mu \mathrm{m}^{2}\right)$ per unit volume of ferrite; $K_{3}$ is a constant, and according to the Chipman experimental results, $K_{3} S_{\alpha}=10.45+2.5 \cdot(1000 / \mathrm{T}) \cdot 44.6$, $[12,13] ; N_{\mathrm{A}}^{\langle i\rangle}$ is the nucleation rate of austenite in the $\mathrm{i}$-th iterative step; $G_{A}^{\langle i\rangle}$ is the austenite growth rate in the $i$-th iteration step, which can be expressed as:

$$
\left\{\begin{array}{l}
N_{A}^{\langle i\rangle}=\frac{K_{1} D_{\mathrm{C}}^{\langle i\rangle}}{\left(k T^{\langle i\rangle}\right)^{1 / 2}} \exp \left(-\frac{K_{2}}{\mathrm{k} T^{\langle i\rangle} \Delta G_{A}^{\langle i\rangle^{2}}}\right) \\
G_{A}^{\langle i\rangle}=\frac{D_{C}^{\langle i\rangle}}{4 r_{0}^{\langle i\rangle}} \frac{C_{\gamma \alpha}^{\langle i\rangle}-C_{\alpha}^{\langle i-1\rangle}}{C_{\alpha}^{\langle i-1\rangle}-C_{\alpha \gamma}^{\langle i\rangle}}
\end{array}\right.
$$

$$
C_{\alpha}^{\langle i-1\rangle}=\frac{C_{\alpha}^{0}}{1-X_{A}^{\langle i-1\rangle}}
$$

where $K_{1}$ and $K_{2}$ are material-dependent coefficients, which are respectively $3.17 \times 10^{3} \mathrm{~J}^{3} / \mathrm{mol}^{3}$ and $4.25 \times 10^{9}$ $\mathrm{J}^{3} / \mathrm{mol}^{3}, k$ is the Pertzman constant. $r_{0}$ is the austenite phase limit radius of curvature, $1.8 \times 10^{-6} \mathrm{~cm}, C_{\gamma \alpha}^{\langle i\rangle}$ and $C_{\alpha \gamma}^{\langle i\rangle}$ are the equilibrium molar fractions of the $\gamma$ and $\alpha$ sides at the $\gamma / \alpha$ phase boundary. $C_{\alpha}^{0}$ is the original $\mathrm{C}$ content in the $\alpha$ phase, respectively, and are calculated by the phase transition KRC phase transition heat model $[14,15]$.

\subsection{Austenite Grain Size Model}

Based on the classical nucleation and growth theory, the grain size of austenite is heated. It is assumed that the new phase austenite grows along the ferrite grain boundary and grows in the shape of an ellipsoid, and has nothing to do with the nucleation rate and time.

In the process of heating austenite reverse phase transformation, the grain size change of austenite consists of two parts, which can be expressed as

$d_{A}=d_{A 1}+d_{A 2}$

where, $d_{A 1}$ is $\gamma$ grain size $(\mu \mathrm{m})$ of heating phase; $d_{A 2}$ is $\gamma$ grain size $(\mu \mathrm{m})$ of heat preservation phase.

In the heating phase, the reverse phase transformation of austenite is phase change in the manner of nucleation growth. According to Cahn's phase transition kinetic theory, the total number of nucleation grains of austenite at the grain boundary of unit volume is expressed as

$$
\begin{aligned}
& n_{A}=\int_{0}^{t_{C}} N_{A}\left(1-X_{A 1}\right) \mathrm{d} t \\
& t_{c}=\frac{T_{f}-A_{s}}{V_{h}}
\end{aligned}
$$

where $t_{c}$ is the time (s) required for the phase transition; $T_{f}$ is the temperature $(\mathrm{T})$ of the heat preservation phase; $A_{S}$ is the starting temperature (T) of austenite reverse phase transition; $N_{A}$ is the growth rate of austenite, $V_{h}$ is the heating rate $(\mathrm{T} / \mathrm{s})$.

Since the $\gamma$ grains are nucleated on the parent phase grain boundaries in an ellipsoidal shape [16, 17], the average grain size of the formed $\gamma$ phase can be expressed as

$d_{A 1}=\left(\frac{6 X_{A 1}^{C}}{\pi n_{A}^{C} S_{\alpha}}\right)^{1 / 3}$

In the heat preservation stage, it is considered that the nucleation position on the grain boundary of the mother phase is saturated. At this stage, the austenite reverse phase transformation is carried out in a phase-saturation phase change mode, only the growth of the crystal grains is calculated, and the shape of austenite is ignored. Nuclear 
process, increase in austenite grain size growth:

$$
d_{A 2}=\int_{t_{C}}^{t_{f}} G_{A}\left(1-X_{A 1}-X_{A 2}\right) \mathrm{d} t
$$

\section{CALCULATION RESULTS}

\subsection{Austenite begins to Change Temperature}

The austenite begins to change temperature $A_{S}$ of the cold-rolled low carbon steel with different deformation amounts to the austenite is shown in Fig. 1. It can be seen from the figure that the heating rate has a great influence on the austenite transformation temperature $A_{S}$, and the $A_{S}$ increases with the heating rate. When the heating speed is less than $5{ }^{\circ} \mathrm{C} / \mathrm{s}$, the heating speed has little effect on the $A_{S}$. With the speed increasing, the phase change onset temperature rises sharply. When the heating rate increases to a certain extent, the phase change onset temperature will tend to a constant value. The influence of deformation on $A_{S}$ decreases with the increase of heating rate. When the heating rate is low (less than $5{ }^{\circ} \mathrm{C} / \mathrm{s}$ ), the deformation amount is $84.6 \%$, and the austenite transformation temperature $A_{S}$ is about $730{ }^{\circ} \mathrm{C}$.

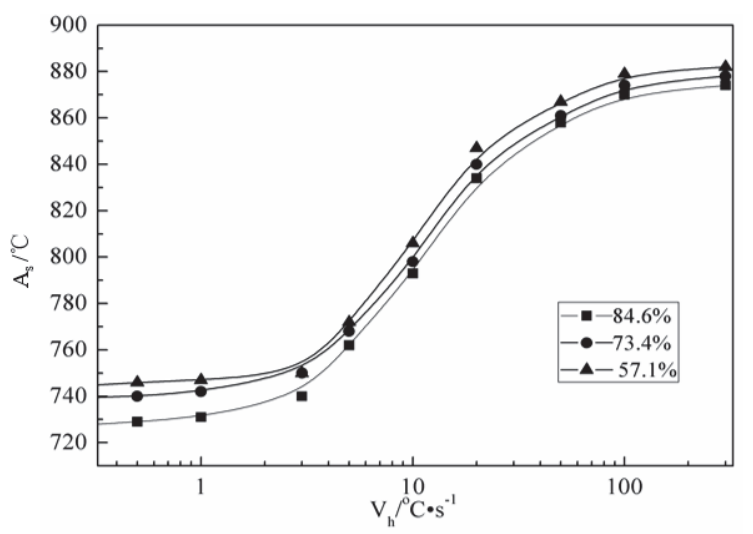

Figure 1 Variation of $A_{s}$ temperature with heating rate in different deformation

The heating rate increases, delaying the occurrence of austenite reverse phase transformation. This is because the reverse phase transformation of heated austenite is a diffusion process. The heating rate directly affects the diffusion ability of the atom. When the heating rate is relatively large, the atom does not have enough time to diffuse, and the relationship between the driving force and the energy is small at this time. When the heating rate exceeds $300{ }^{\circ} \mathrm{C} / \mathrm{s}$, the atomic diffusion is very small, and the transformation of the face centered cubic in the body direction of the cutting deformation occurs directly.

\subsection{Influence of Deformation on Austenite Structure}

Fig. 2 shows the austenite reverse phase transformation, the austenite transformation amount and grain size change with deformation and temperature after annealing and cold rolling deformation. It can be seen from Fig. 2a that at the same temperature, $X_{A}$ increases with the increase of cold rolling deformation and increases with the increase of heating temperature. When the heating temperature is lower than $800{ }^{\circ} \mathrm{C}$, the influence of the deformation amount $X_{A}$ is not obvious, and when the heating temperature is above $830^{\circ} \mathrm{C}, X_{A}$ increased sharply with the increase of the deformation amount, when the deformation amount is about $92 \%$, the austenite transformation amount reached $89.5 \%$.

This is mainly because the deformation amount is increased, the phase change driving force and the nucleation driving force are increased, and the phase transition temperature is advanced, and therefore, the phase transformation rate is increased. In Fig. $2 b$, the grain size $d_{A}$ of austenite decreases with the increase of deformation, when the deformation is more than $90 \%$, the ferrite grain size is less than $5 \mu \mathrm{m}$, and the ultrafine structure is formed.

This is because the deformation not only increases the position of the nucleus of ferrite recrystallization, but also promotes the deformation induced phase transition.

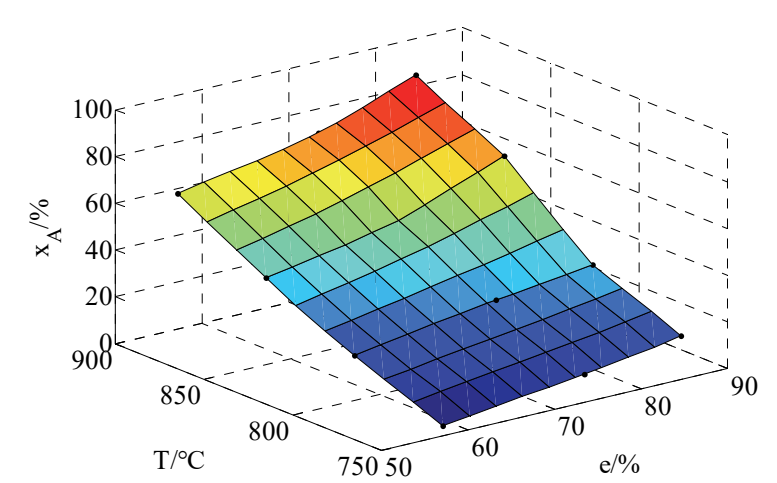

a) $X_{A}$ varies with temperature in different deformation

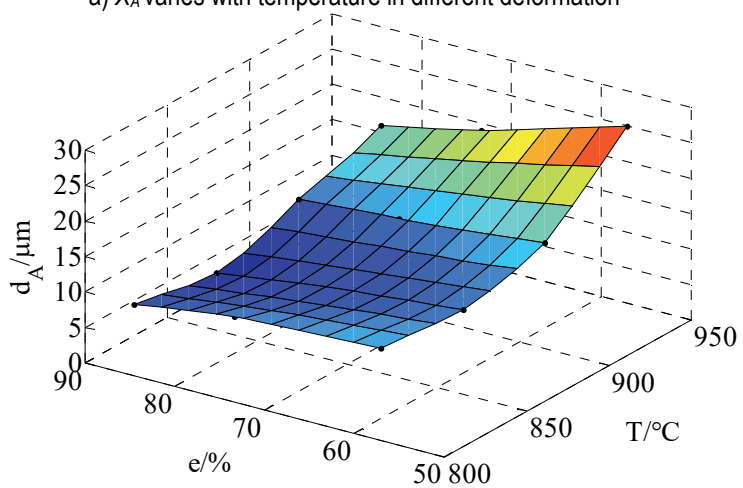

b) $d_{A}$ varies with temperature

Figure 2 Variation of austenitic microstracture in different deformation $\left(V_{h}=10\right.$ $\left.{ }^{\circ} \mathrm{C} / \mathrm{s}, t=30 \mathrm{~s}\right)$

The new phase austenite is in the parent phase. The nucleation at the grain boundary of the ferrite increases the nucleation position of the austenite, so $d_{A}$ decreases as the amount of deformation increases.

\subsection{Effect of Temperature on Austenite Structure}

Fig. 3 shows the variation of the transformation amount and grain size of austenite formed under different heating conditions. As can be seen from Fig. 3a, $X_{A}$ decreases with the increase of heating speed and increases with the increase of heating temperature. When the heating speed is less than $10^{\circ} \mathrm{C} / \mathrm{s}, X_{A}$ has an approximate linear relationship with the heating temperature.

As can be seen from Fig. 3b, the grain size grows rapidly with the increase of temperature at the same time. When the temperature is less than $900{ }^{\circ} \mathrm{C}$, the holding time has no obvious effect on the grain growth. The heating temperature increased from $1000^{\circ} \mathrm{C}$ to $1050{ }^{\circ} \mathrm{C}$, the grain size increased to more than $40 \mu \mathrm{m}$, forming coarse 
structure.

With the prolongation of the heat holding time, the crystal grains grow gradually. Under the premise that the formation time of the heated austenite is short, the temperature is the decisive factor affecting the austenite grain size.

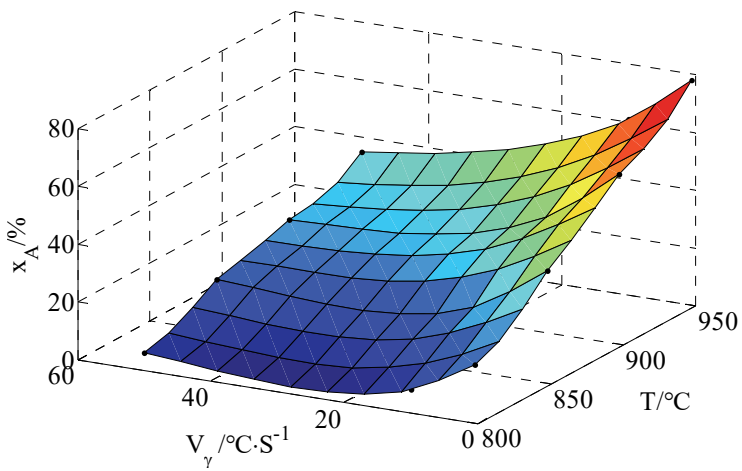

a) The three-dimensional relationship between $X_{A}$ with heating speed and temperature

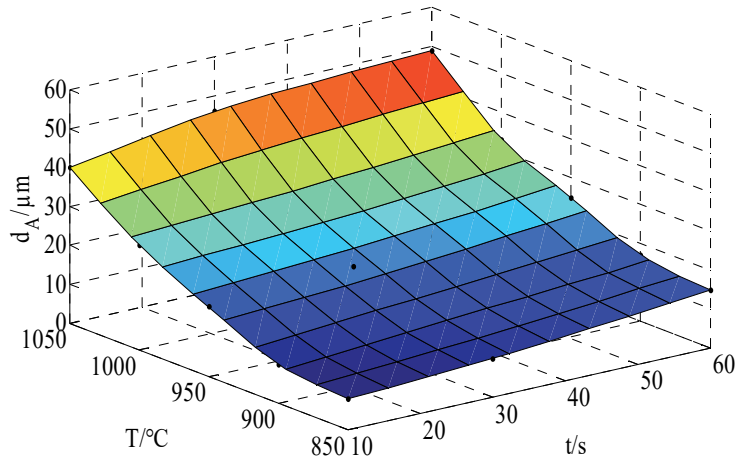

b) The three-dimensional relationship between $d_{A}$ with heating temperature and time

Figure 3 Variation of austenitic microstracture in different heating process

\section{EXPERIMENTAL SCHEME AND RESULTS VERIFICATION}

\subsection{Experimental Materials}

The experimental material used was a hot rolled sheet SPHC produced by CSP, and its chemical composition is shown in Tab. 1, and the thickness of the sheet was $3.5 \mathrm{~mm}$.

Table 1 Chemical composition of the test steel (wt / \%)

\begin{tabular}{|c|c|c|c|c|c|}
\hline $\mathrm{C}$ & $\mathrm{Si}$ & $\mathrm{Mn}$ & $\mathrm{S}$ & $\mathrm{P}$ & $\mathrm{Al}$ \\
\hline 0.05 & 0.03 & 0.35 & 0.02 & 0.02 & 0.03 \\
\hline
\end{tabular}

\subsection{Experimental Process}

The material was processed into 10 strips $40 \mathrm{~mm}$ wide, and the sample numbers were: $0,1,2, \ldots, 8,9$. In the tworoll reversing mill, multiple passes were cold-rolled and deformed, and the cumulative deformation was $0 \%, 18.6 \%$, $28.6 \%, 39.2 \%, 43.7 \%, 57.1 \%, 66.3 \%, 73.4 \%, 79.2 \%$, $84.6 \%$. The rolled sheet was polished, polished and cleaned to prepare a sample of $h \times 35 \times 25 \mathrm{~mm}$. The austenite reverse phase transformation simulation experiment was carried out on a Gleeble-3500 thermal simulation test machine. The experimental process route is shown in Fig. 4. The sample was heated to the experimental temperature at a rate of $20{ }^{\circ} \mathrm{C} / \mathrm{s}$ for a certain period of time, and then quenched by water quenching. The austenite zone heating temperature is $930{ }^{\circ} \mathrm{C}$ and $1000^{\circ} \mathrm{C}$, and the heat holding time is $20 \mathrm{~s}, 1 \mathrm{~min}$ and $5 \mathrm{~min}$. The austenitic ferrite twophase zone has heating temperatures of $750{ }^{\circ} \mathrm{C}, 800{ }^{\circ} \mathrm{C}$, $850^{\circ} \mathrm{C}$ and $900{ }^{\circ} \mathrm{C}$, and an isothermal time of $20 \mathrm{~s}$

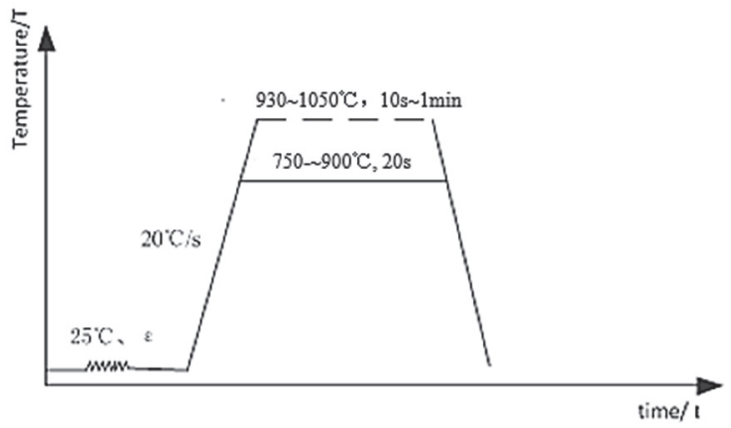

Figure 4 Schematic experimental process of heat treating

\subsection{Experimental Verification}

Fig. 5 is an austenite structure formed by cold-rolled materials of different deformation amounts at $950{ }^{\circ} \mathrm{C}$ for 30 s. From Fig. 5a, it can be calculated that the average grain size is $36 \mu \mathrm{m}$. By comparing Fig. 5a with Fig. 5b, it can be seen that the difference in average grain size is very small.

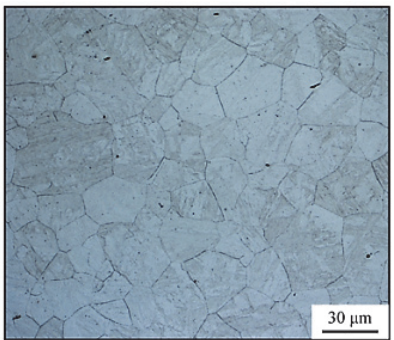

a) $18.6 \%$

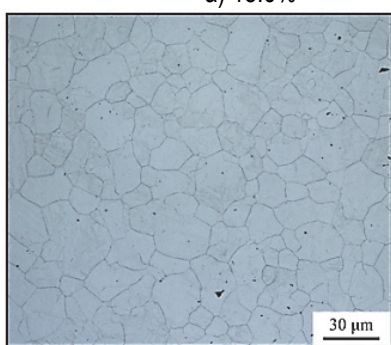

c) $73.4 \%$

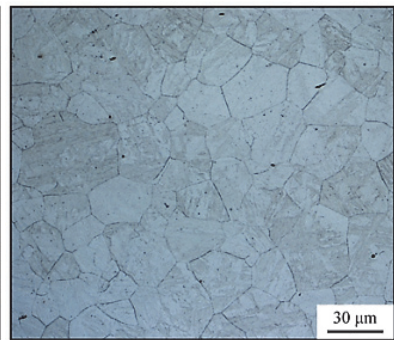

b) $39.2 \%$

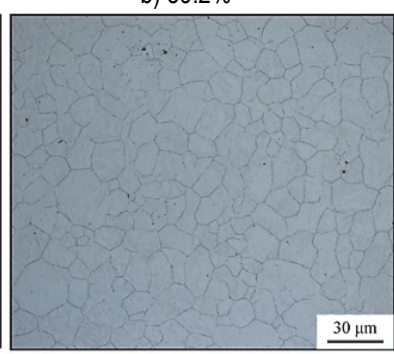

d) $84.6 \%$
Figure 5 austenitic structures when heating in different deformation

As the deformation amount increases, the austenite grains are refined, and when the deformation amount is $84.6 \%$, the grain refinement is $22 \mu \mathrm{m}$. As can be seen from Figs. 5a and $5 \mathrm{~d}$, when the deformation amount is large and small, the austenite grains formed are relatively uniform. Observe Figs. $5 \mathrm{~b}$ and $5 \mathrm{c}$ the austenite grain size is not uniform when the deformation amount is $39 \%$ to $73 \%$.

Cold rolling deformation has an important influence on the formation of austenite. Cold rolling causes severe lattice distortion inside the material, which provides energy fluctuation and structural fluctuation for the nucleation of austenite. The larger the deformation amount is, the higher nucleation rate of austenite is, and the finer the austenite grains formed. When the amount of deformation is small, the deformation has little effect on the formation of austenite. In the case of moderate deformation, only a part 
of the austenite nucleates at the deformation zone, and the austenite grains are formed to grow rapidly, so that the austenite grain size is not uniform.

Fig. 6 shows the austenite structure generated by different heating processes. It can be seen from the Fig. $6 \mathrm{a}$ that the initial grain size of austenite is $14 \mu \mathrm{m}$; there is a small amount of unconverted ferrite at the equiaxed austenite grain boundary, and the austenite is not completely transformed. Comparing the Figs. $6 \mathrm{~b}$ and $6 \mathrm{~d}$, it can be seen that the grain grows obviously when the temperature increases by $100^{\circ} \mathrm{C}$.

As can be seen from Fig. 6d, after holding time at 1050 ${ }^{\circ} \mathrm{C}$ for $1 \mathrm{~min}$, the average grain size is about $42 \mu \mathrm{m}$. The Figs. $6 \mathrm{c}$ and $6 \mathrm{~d}$ show that after holding at $1050{ }^{\circ} \mathrm{C}$ for 30 $\mathrm{s}$, the grain grows and the size is not uniform as the heat holding time is extended.

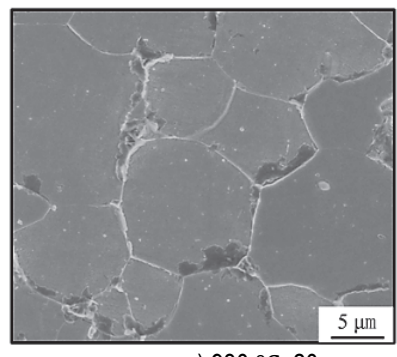

a) $930{ }^{\circ} \mathrm{C}, 20 \mathrm{~s}$

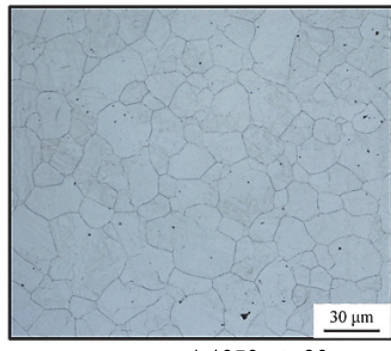

c) $1050^{\circ} \mathrm{C}, 30 \mathrm{~s}$

Figure 6 Effect of heating technology on austenitic structure

In addition, there are severe lattice distortions inside the cold-rolled strip, severe grain deformation, and uneven deformation affects the austenite grains formed. The austenite grains formed by nucleation are prone to be long in the case of severe lattice distortion. The formation of large crystal grains increases the grain size unevenness with time and temperature. In order to avoid overheating and austenite grain size non-uniformity, the heating temperature should be reduced as much as possible and the heat holding time should be shortened.

\section{CONCLUSION}

1) Considering the phase change energy storage, a model of incubation period describing the austenite transformation during heating is established, and the phase variable model and grain size model of the austenite structure are formed;

2) The austenite phase transition temperature $A_{S}$ increases with the heating rate and approaches a constant value. The effect of deformation on $A_{S}$ decreases with the increase of heating rate. When the heating rate is low (less than $5{ }^{\circ} \mathrm{C} / \mathrm{s}$ ), the deformation amount is $84.6 \%$, austenite start transition temperature $A_{S}$ is about $730{ }^{\circ} \mathrm{C}$;

3) $X_{A}$ increases with the increase of cold rolling deformation, $d_{A}$ decreases with the increase of deformation, austenite grain size grows rapidly with the increase of temperature, $A_{S}$ the amount of deformation increases, the austenite grains are refined, and the average grain size is about $22 \mu \mathrm{m}$ when the deformation is $84.6 \%$. The experimental calculation shows that the model is accurate and the error rate is less than $10 \%$. So, it is of great significance for the microstructure controlling and process optimization of cold rolling low carbon steel.

\section{Acknowledgement}

The Talented Projects of Quzhou University (BSYJ201402) and the Talented Projects of Quzhou University (BSYJ201403).

\section{REFERENCES}

[1] Pei, X. H., Liu, Z. Y., Wie, J. et al. (2018). Microstructure and Properties of a Low Carbon Ti-V Microalloyed Steel. Journal of Wuhan University of Technology (Materials Science), 33(06), 1491-1495. https://doi.org/10.1007/s11595-018-1996-4

[2] Timokhina, I. B., Hodgson, P. D., \& Pereloma, E. V. (2003). Effect of deformation schedule on the microstructure and mechanical properties of a thermo mechanically processed $\mathrm{C}-\mathrm{Mn}-\mathrm{Si}$ transformation-induced plasticity. Metallurgical and Materials Transactions A, 34(8), 1599-1609. https://doi.org/10.1007/s11661-003-0305-8

[3] Zhou, Z. A., Fu, W. T., Zhu, Z. et al. (2018). Excellent mechanical properties and resistance to cavitation erosion for an ultra-low carbon $\mathrm{Cr} \mathrm{Mn} \mathrm{N}$ stainless steel through quenching and partitioning treatment. International Journal of Minerals Metallurgy and Materials, 25(05), 547-553. https://doi.org/10.1007/s12613-018-1601-z

[4] Chong, Y., Liu, Z. D., Wang, L. et al. (2015). Heat Treatment of a candidate material for $700{ }^{\circ} \mathrm{C}$ A-USC power plants. Journal of Iron and Steel Research (International), 22(02), 150-156. https://doi.org/10.1016/S1006-706X(15)60023-1

[5] Li, P., Li, J., Meng, Q. G. et al. (2015). Influence of rapid heating process on the microstructure and tensile properties of high-strength ferrite-martensite dual-phase steel. International Journal of Minerals Metallurgy and Materials, 22(09), 933-941. https://doi.org/10.1007/s12613-015-1152-5

[6] Gomez, M., Medina, S. F., \& Caruana, G. (2003). Modelling of Phase Transformation Kinetics by Correction of Dilatometry Results for a Ferritic Nb-Microalloyed Steel. ISIJ International, 43(8), 1228-1237. https://doi.org/10.2355/isijinternational.43.1228

[7] Zhu, Y. T., Lowe, T. C., \& Asaro, R. J. (1997). Assesment of the theoretical basis of the rule of additivity for the nucleation incubation time during continuous cooling. Journal of Applied Physics, 82(03), 1129. https://doi.org/10.1063/1.365879

[8] Liu, Z. Y., Xu, Y. B., \& Wang G. D. (2004). Simulation and prediction of the evolution of microstructure and propreties of hot rolled steels. Northeastern University Press, Shenyang.

[9] Pham, M. S., Solenthaler, C., Janssens, K. G. F. et al. (2011). Dislocation Structure Evolution and its Effects on Cyclic Deformation Response of Aisi 316L Stainless Steel. Materials Science and Engineering A, 528(7-8), 3261-3269. https://doi.org/10.1016/j.msea.2011.01.015

[10] Adams, B. L., Gardner, C. J., \& Fullwood, D. T. (2009, September 23-25). Ebsd-Based Dislocation Microscopy. $3^{\text {rd }}$ International Conference on Texture and Anisotropy of Polycrystals, ITAP-3.

[11] Zhuang, L., Di, W., Hui, Z. et al. (2011). Influence of Controlled Rolling and Cooling Process on the Mechanical 
Properties of Low Carbon Cold Forging Steel. Journal of Iron and Steel Research (International), 18(S1), 595-599.

[12] Xu, Z. Y. (2005). Materials Thermodynamics. Science Press, Beijing.

[13] Song, S. J., Liu, F., \& Jiang, Z. H. (2014). Generalized additivity rule and isokinetics in diffusion controlled grow. Journal of Materials Science, 49(06), 2624-2629. https://doi.org/10.1007/s10853-013-7964-7

[14] Peng, N. Q., Tang, G. B., Liu, Z. D. et al. (2009). Modification of zener's two-parameter in the superelement model for Fe- $\sum \mathrm{X}_{\mathrm{i}}-\mathrm{C}$ alloy systems. Acta Metallurgica Sinica, 45(03), 331-337.

[15] Tanaka, T., Aaronson, H. I., \& Enomoto, M. (1995). Calculation of $\alpha / \gamma$ phase boundaries in Fe-C-X $1-\mathrm{X}_{2}$ systems from the central atoms model. Metallurgical Transactions A, 26(03), 535-545. https://doi.org/10.1007/BF02663904

[16] He, L. F., Li, H. P., Gai, K. et al. (2015). Phasetransformation kinetics of austenization for 55CrMo steel. Transactions of Materials and Hest transactions of Materials and Heat Treatment, 36(10), 255-260.

[17] Hashemi, S. G. \& Eghbali, B. (2018). Analysis of the formation conditions and characteristics of interphase and random vanadium precipitation in a low-carbon steel during isothermal heat treatment. International Journal of Minerals Metallurgy and Materials, 25(03), 339-349. https://doi.org/10.1007/s12613-018-1577-8

\section{Contact information:}

\section{Su-fen WANG}

Key Laboratory of Air-drive Equipment Technology of Zhejing, Province Quzhou, Zhejiang 324000, P. R. China

Quzhou University,

NO. 78 Jiuhua North Road, Quzhou, Zhejiang 324000, China

\section{Zhi-jie LI}

(Corresponding author)

Key Laboratory of Air-drive Equipment Technology of Zhejing

Province Quzhou, Zhejiang 324000, P. R. China

Quzhou University,

NO. 78 Jiuhua North Road, Quzhou, Zhejiang 324000, China

\section{Jian-hong HUANG}

SHANG YANG

Machinery Co., Ltd of Zhejiang

Quzhou, Zhejiang 324000, P. R. China

\section{Xu-yang CHEN}

College of Mechanical Engineering,

Quzhou University,

Quzhou, Zhejiang 324000 , P. R. China

NO. 78 Jiuhua North Road, Quzhou, Zhejiang 324000, China

\section{Xin-dong WANG}

College of Mechanical Engineering,

Quzhou University,

Quzhou, Zhejiang 324000 , P. R. China

NO. 78 Jiuhua North Road, Quzhou, Zhejiang 324000 , China 